Okocha EC

Ulasi T

Aneke JC

Ajuba IC

Okwummuo EP

\section{Unusual presentations of childhood acute lymphoblastic leukaemia: A case report}

DOI:http://dx.doi.org/10.4314/njp.v41i3,18

Accepted: 6th April 2014

Aneke JC ( )

Okocha EC, Ajuba IC, Okwummuo EP

Department of Haematology,

Ulasi T

Department of Paediatrics,

Nnamdi Azikiwe University Teaching

Hospital, PMB, 5025, Nnewi,

Anambra State, Nigeria.

Email: anekejc@ymail.com.

\begin{abstract}
Childhood acute lymphoblastic leukaemia, (ALL) is increasingly reported to present in an atypical fashion which may have significant implications for treatment outcomes and survival. This case report presents a

Nigerian child who's clinical and radiological features together with effusion cytological findings were suggestive of metastatic neuroblastoma. However, a definitive diagnosis of ALL was established
\end{abstract}

following a bone marrow aspiration study that revealed abnormal cellularity consistent with L1 morphological subtype. Unfortunately, the child was discharged against medical advice before definitive therapy could be commenced.

Key words: Atypical presentations, metastatic neuroblastoma, childhood acute lymphoblastic leukaemia.

\section{Introduction}

Acute lymphoblastic leukaemia (ALL) is a malignant haematological condition that arises from an acquired somatic mutation in a lymphoid progenitor cell ${ }^{1}$. This mutation may occur at various points in the development of the lymphoid progenitor. Malignant proliferation and accumulation of lymphoid blasts in the bone marrow and some extramedullary sites such as the liver, spleen, skin, testes (in males) and even the central nervous system (CNS) is the hallmark of this disease.

ALL accounts for up to $30 \%$ of childhood cancers in Caucasians $^{2}$, thus it is among the most common paediatric malignancies.

Clinically, ALL may have diverse patterns of presentation; typically its clinical presentation is related to bone marrow failure and extra medullary effects of the disease. As such common symptoms range from those arising secondary to cytopaenias (including anaemia, leucopaenia and thrombocytopaenia) to those due to organ/ system infiltration such as lymphadenopathy and hepatosplenomegaly. Increasingly, unusual presentation of childhood ALL is being documented in literature and cases presenting with back pain and vertebral compres$\operatorname{sion}^{3}$, stroke $^{4}$, absence of blasts in the peripheral blood ${ }^{5}$, obstructive jaundice ${ }^{6}$, and isolated masseter muscle involvement ${ }^{7}$ have been variously reported.

We report here a case of ALL presenting with atypical features, in a Nigerian child to highlight challenges of diagnosis.

\section{Case Report}

A 4- year- old male Nigerian was seen at the Nnamdi Azikiwe University Teaching Hospital, Nnewi, with a 14- week history of recurrent fever, multiple facial swellings with enlargement of the head and protrusion of the eyes, (fig. 1). There was also a history of significant weight loss and nasal discharge that occasionally was blood tinged.

Fig1: Showing head and anterior chest wall swellings

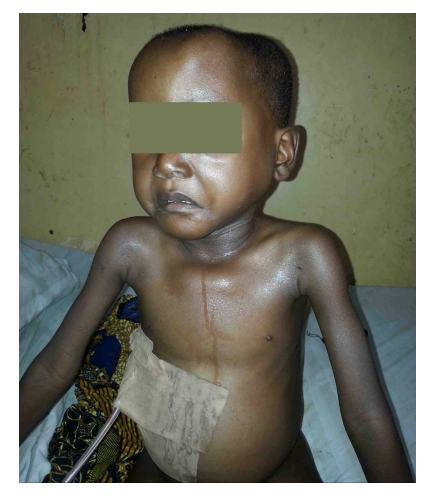

General examination was significant for marked weight loss, moderate mucosal pallor, significant generalized lymph node enlargement and bipedal pitting oedema. Three discrete masses were noted on the left part of the frontal bone, left part of the jaw and anterior chest wall, measuring $10 \mathrm{~cm}, 6 \mathrm{~cm}$ and $9 \mathrm{~cm}$ in their longest diameters respectively. These swellings were globular, firm to hard in consistency, non mobile, non tender and were neither attached to overlying skin nor showed any differential warmth.

Bilateral parietal bossing along with coronal sutural diathesis was also noted. The anterior fontannel was 
patent and normotensive, measuring $2 \mathrm{~cm} \times 2 \mathrm{~cm}$. He had no signs of meningeal irritation and muscle tone and power were normal globally.

His abdomen was uniformly distended with palpably enlarged, firm and tender liver, $10 \mathrm{~cm}$ below the right costal margin. Ascites was present and demonstrable.

Chest examination was significant for reduced chest expansion and stony dull percussion notes over the right hemi thorax with absence of breath sounds in both the right mid and lower zones.

A provisional clinical diagnosis of neuroblastoma metastatic to the right hemi thorax and the head region was considered.

An abdomino-pelvic ultrasonographic examination showed a right sided supra renal mass while a chest radiograph demonstrated right sided pleural effusion, the cytology of which revealed hypercellular smears showing sheets of medium sized cells with high nucleocytoplasmic ratio, in a dirty background. The neoplastic cells have coarse chromatin pattern. Overall features were suggestive of a malignant (round) blue cell tumour, probably neuroblastoma. These findings reinforced metastatic neuroblastoma as the most probable diagnosis. The patient was seronegative for HIV 1 and 2, while haemoglobin electrophoresis confirmed AA haemoglobin phenotype. Complete blood count was significant for severe anaemia (Haematocrit was $0.17 \mathrm{~L} / \mathrm{L}$ ), moderate leucocytosis (white cell count of $20.8 \times 10^{9} / \mathrm{L}$ ) and mild thrombocytopaenia (platelet count of $76 \times 10^{9} / \mathrm{L}$ ). Blood film and bone marrow cytology were however in keeping with ALL, L1 morphological type (figs 2 and 3). Flow cytometric analysis of peripheral blood cells showed positivity for CD 45, an extended immunophenotypic profile as well as cranial computed tomography (CT) scan could not be done because of the non availability of funds.

Fig 2: Bone marrow film, showing L1 lymphoblasts

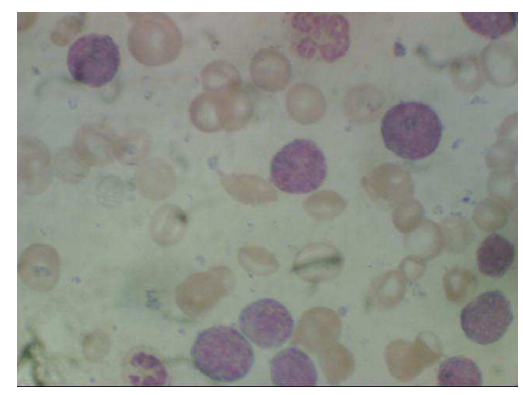

Fig 3: Peripheral blood film, showing lymphoblasts

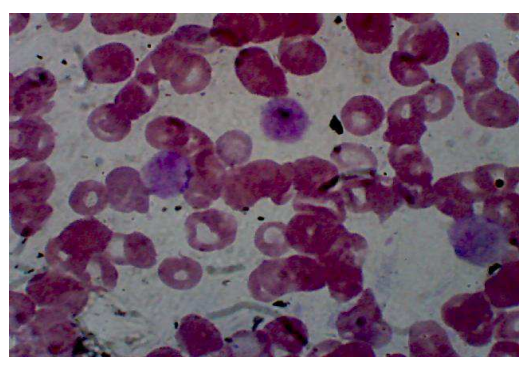

Supportive treatment including blood product transfusions were commenced while work up including biopsy of the masses was planned as a prelude to definitive chemotherapy for ALL. However, further care was abruptly terminated as child was discharged against medical advice.

\section{Discussion}

Atypical presentations of ALL have reportedly constituted an enormous challenge, in terms of diagnosis, especially in resource poor settings. However, advances in diagnostic protocols, especially in the realm of immunophenotyping and relevant molecular diagnostics have greatly enhanced diagnostic precision in such atypical cases $^{3-6}$. While such levels of diagnostic accuracy is desirable, the application of basic cytological techniques for the analysis of appropriate specimens has continued to provide valuable information in resource poor settings. In this patient, bone marrow study was able to establish a diagnosis of ALL.

Our patient presented with head enlargement, facial and anterior chest wall swellings. Typically children with ALL tend to present with extra medullary organ enlargement owing to infiltration by lymphoblasts and while the liver, spleen and testes are the most frequently affected organs, other less common extra medullary sites have been reported in the literature. Coronal sutural diathesis as well the patent anterior frontannel observed in this patient may be an indication of CNS infiltration by lymphoblasts; a CT scan however was not done to confirm this. Wimperis et $\mathrm{al}^{7}$ in 1992 described two children with ALL in whom isolated masseter muscle involvement was the only presenting feature of the disease. Accurate diagnoses of the cases were hinged on the use of immunophenotyping and immunogenotyping. Indeed, such extended panel of diagnostic tools have proved useful in establishing diagnoses in similar atypical cases of ALL presenting as vertebral compression ${ }^{3}$, stroke $^{4}$, absence of blasts in peripheral blood ${ }^{5}$ and obstructive jaundice ${ }^{6}$.

The initial diagnosis in this child was metastatic neuroblastoma based on his age and the clinical presentation of multiple masses in the head region and chest and reenforced by the ultrasonographic finding of a supra renal mass together with the pleural effusion cytology report. However, in this patient, peripheral blood and bone marrow cytology were both in keeping with ALL of the L1 morphological type. Besides, the demonstration of CD 45 lineage antigen supported the haematopoietic origin of this malignant condition. In a recent case study, D'angelo et al, ${ }^{8}$ reported ALL co-existing with neuroblastoma in a 3 year old girl, as different disease entities. We had entertained the possibility of this phenomenon at a stage in the management of this child prior to bone marrow investigations; in point of fact, biopsied tissue sample of one of the masses had been scheduled but later considered unnecessary.

Advances in ALL treatment have ushered in an individualized, tailored and risk adapted approach, utilizing a myriad of chemotherapy options with or without haematopoietic stem cell transplantation. Treatment 
stratification is commonly based on the biologic features of individual disease ${ }^{2}$. Utilizing the risk adapted protocol, Pui et al, ${ }^{9}$ suggested that prophylactic cranial irradiation, which has been a component of the standard treatment of childhood ALL, may safely be omitted. Unfortunately our patient did not stay long enough in our care to receive any definitive treatment; he was not followed up to the community.

\section{Conclusion}

Childhood ALL may present in a rather atypical manner. A high index of suspicion, complimented by appropriate diagnostic tests is essential in making early and accurate diagnoses in cases of ALL with atypical presentations.

\section{Conflict of Interest: None \\ Funding: None}

\section{Limitation of this report}

A biopsy and histology of the body masses, including that on the adrenals (preferably via ultrasound guide) might have been a more definite way to rule out the possibility of neuroblastoma co-existing with ALL in this child, this was however not done.

\section{References}

1. Provan D, Singer CRJ, Baglin T, Dokal I. Oxford Handbook of Clinical Haematology. Oxford University Press, New York. $3^{\text {rd }}$ Ed. 2009.p132.

2. Lo Nigro L. Biology of childhood acute lymphoblastic leukaemia. $J$ PediatrHematol Oncol. 2013;35:245-52.

3. Hafiz MG, Islam A, Siddique R. Back pain and vertebral compression: an unusual presentation of childhood acute lymphoblastic leukaemia. Mymensingh Med J 2010; 19:130-6.

4. Ege MJ, Meyer LH, Debatin KM, Stahnke K. Co-incidence of recurrent hemiparesis and detection of acute lymphoblastic leukaemia in a 4 year old girl: one or two disease. KlinPediatr. 2009;221:3869.
5. Cogulu O, Karapinar DY, Karaca E, Aydinok Y, Ozkinay F. Unusual course of an acute lymphoblastic leukaemia case with i (9q) as a sole cytogenetic abnormality. Leuk Res. 2006; 30:14613 .

6. Alvaro F, Jain M, Morris LL, Rice MS. Childhood acute lymphoblastic leukaemia presenting with jaundice. J Paediatr Child Health. 1996;32:466-8.

7. Wimperis JZ, Brandt LJ, O'Connor S, Marcus R, Broadbent V. Unusual presentation of common acute lymphoblastic leukaemia antigen-positive extra medullary disease in childhood. Two patients with isolated masseter muscle involvement. Cancer. 1992 15;70:8897-901.
8. D'angelo, Grigoli A, Sementa AR, Tropia S, Alaggio R, Arico M. Simultaneous diagnosis of acute lymphoblastic leukaemia and peripheral neuroblastic tumour in a child. J PediatrHematol Oncol. 2012;34:75-5.

9. Piu CH, Campana D, Pei D, Bowman WP, Sandlund JT, Kaste SC et al. Treating childhood acute lymphoblastic leukaemia without cranial irradiation. $N$ Engl J Med. 2009;360:2730-41. 